JOURNAL OF THE

AMERICAN MATHEMATICAL SOCIETY

Volume 13, Number 3, Pages 595-609

S 0894-0347(00)00333-7

Article electronically published on March 15, 2000

\title{
EXPLICIT QUANTIZATION OF DYNAMICAL R-MATRICES FOR FINITE DIMENSIONAL SEMISIMPLE LIE ALGEBRAS
}

\author{
PAVEL ETINGOF, TRAVIS SCHEDLER, AND OLIVIER SCHIFFMANN
}

\section{INTRODUCTION}

1.1. Classical r-matrices. In the early eighties, Belavin and Drinfeld $\mathrm{BD}$ classified nonskewsymmetric classical $r$-matrices for simple Lie algebras. It turned out that such r-matrices, up to isomorphism and twisting by elements from the exterior square of the Cartan subalgebra, are classified by combinatorial objects which are now called Belavin-Drinfeld triples. By definition, a Belavin-Drinfeld triple for a simple Lie algebra $\mathfrak{g}$ is a triple $\left(\Gamma_{1}, \Gamma_{2}, T\right)$, where $\Gamma_{1}, \Gamma_{2}$ are subsets of the Dynkin diagram $\Gamma$ of $\mathfrak{g}$, and $T: \Gamma_{1} \rightarrow \Gamma_{2}$ is an isomorphism which preserves the inner product and satisfies the nilpotency condition: if $\alpha \in \Gamma_{1}$, then there exists $k$ such that $T^{k-1}(\alpha) \in \Gamma_{1}$ but $T^{k}(\alpha) \notin \Gamma_{1}$. The r-matrix corresponding to such a triple is given by a certain explicit formula. These results generalize in a straightforward way to semisimple Lie algebras.

In [S], the third author generalized the work of Belavin and Drinfeld and classified classical nonskewsymmetric dynamical r-matrices for simple Lie algebras. It turns out that they have an even simpler classification: up to gauge transformations, they are classified by generalized Belavin-Drinfeld triples, which are defined as the usual Belavin-Drinfeld triples but without any nilpotency condition. The dynamical rmatrix corresponding to such a triple is given by a certain explicit formula. As before, these results can be generalized to semisimple Lie algebras.

1.2. Quantization of $\mathbf{r}$-matrices. The problem of quantization of the BelavinDrinfeld r-matrices (i.e. finding the corresponding quantum R-matrices) has been open for a long time. The history of this problem is as follows.

In the case when $\Gamma_{i}$ are empty (the "standard" r-matrix), the quantization was provided by Drinfeld and Jimbo in the mid 80's, which gave birth to the modern theory of quantum groups.

In 1990, Cremmer and Gervais [CG] provided an explicit quantum R-matrix (in the vector representation) for the so-called Cremmer-Gervais triple for $s l_{n}$ - the Belavin-Drinfeld triple where $\Gamma_{1}$ and $\Gamma_{2}$ are the whole Dynkin diagram without the last and the first vertex, respectively, and $T$ is the shift to the right by one position. Other proofs of the fact that the Cremmer-Gervais R-matrix satisfies the quantum Yang-Baxter equation and the Hecke relation were given by Hodges $\mathrm{H1}$, H2.

In 1992, Gerstenhaber, Giaquinto, and Schack [GGS suggested a conjectural explicit quantization of all Belavin-Drinfeld r-matrices for the Lie algebra $s l_{n}$, in

Received by the editors December 1, 1999 and, in revised form, February 10, 2000.

2000 Mathematics Subject Classification. Primary 17B37.

(C)2000 American Mathematical Society 
the vector representation (the GGS conjecture); it has been checked in many cases GH] Sch1, but a general proof is still unavailable.

In 1995, it was shown in EK that a quantization exists in principle, but the method of [EK] does not allow one to compute the quantization explicitly.

In 1996, Hodges [H3] suggested an explicit quantization in the case when $\Gamma_{1} \cap$ $\Gamma_{2}=\emptyset$ (for any Lie algebra), which yields a proof of the GGS conjecture in this case. Namely, he constructed a twist which transforms the usual quantum group $U_{q}(\mathfrak{g})$ to a quantum group that is a quantization of the Lie bialgebra corresponding to the given Belavin-Drinfeld triple with disjoint $\Gamma_{1}$ and $\Gamma_{2}$.

In early 1999, the second author generalized the method of Hodges to a wider class of triples $(\underline{\mathrm{Sch} 2})$. He also did computer calculations which led him to a conjectural quantization of all triples for $s l_{n}$ in the vector representation. This work led to an understanding of what the generalization of Hodges' formula to any triple should look like, and eventually to a complete explicit solution of the problem, which is given here.

1.3. Description of the paper. In this paper, we provide an explicit quantization of dynamical r-matrices for semisimple Lie algebras, classified in [S], which includes the Belavin-Drinfeld r-matrices. We do so by constructing an appropriate (dynamical) twist in the tensor square of the Drinfeld-Jimbo quantum group $U_{q}(\mathfrak{g})$. The construction of this twist is based on the method stemming from [JKOS] and ABRR, i.e. on defining the twist as a unique solution of a suitable difference equation. This yields a simple closed formula for the twist.

In the case of ordinary Belavin-Drinfeld triples (i.e. satisfying the nilpotency condition), the constructed dynamical twist can be made independent of the dynamical parameter by a gauge transformation. Computing this constant twist and the corresponding R-matrix in the vector representation in the case $\mathfrak{g}=s l(n)$, we obtain an explicit solution of the quantum Yang-Baxter equation on an $n$-dimensional space. This solution is exactly the same as suggested earlier in Sch2 on the basis of computer calculations. The second author conjectured in Sch2 that it coincides with the GGS solution, and checked it for $n \leq 12$ using a computer. This conjecture is also proved in $\mathrm{Sch} 2$ for many special types of Belavin-Drinfeld triples.

Remark 1.1. The new dynamical twists constructed in this paper give rise to new examples of Hopf algebroids (quantum groupoids). There are two methods to construct a Hopf algebroid out of a dynamical twist - the method of [Xu and the method of [EV], and the results they give are, essentially, dual to each other. For nilpotent triples, when the twist is constant (in which case both methods are classical), the first method yields a new quantum deformation of $U(\mathfrak{g})$ as a Hopf algebra, and the second method yields a quantum deformation of the function algebra $\operatorname{Fun}(G)$ on the corresponding group. We expect that the study of the structure and (co)representation theory of these new quantum groups and groupoids is a very interesting and fruitful direction of future research.

Remark 1.2. The results of this paper can be generalized to Kac-Moody algebras, which we plan to do in a forthcoming paper. This generalization is especially interesting in the case of affine Lie algebras. In this case, projecting the obtained quantum dynamical R-matrices to finite dimensional representations, one obtains the quantization of classical dynamical r-matrices with spectral parameters which 
are discussed in [ES]. In the case of the Felder and the Belavin r-matrix, it was done in JKOS, by a method essentially the same as ours.

Remark 1.3. In [ER], the authors defined the notion of a triangular twist, in order to represent quantum determinants of quantum groups as products of quasideterminants. We would like to emphasize that the twist we constructed in this paper is triangular according to Definition 1.2 in [ER]. Therefore, the main theorem of [ER], which claims that the quantum determinant is a product of quasideterminants, is valid for any Belavin-Drinfeld triple, as was anticipated in [ER].

1.4. Contents. In Section 2 we discuss the theory of generalized Belavin-Drinfeld triples and the corresponding dynamical r-matrices.

In Section 3 we give the construction of the twist $\mathcal{J}_{T}$ corresponding to a BelavinDrinfeld triple, state the main result (that it satisfies the dynamical 2-cocycle condition), and show, by computing the quasiclassical limit of $\mathcal{J}_{T}$, that it defines a quantization of the triple.

In Section 4 we prove the 2-cocycle condition for $\mathcal{J}_{T}$.

In Section 5, we quantize gauge transformations for dynamical r-matrices, thus giving an explicit quantization for all the r-matrices considered.

In Section [6] we discuss the nilpotent case, in which the twist can be made nondynamical by a gauge transformation. We compute the explicit form of this twist, which confirms a conjecture of the second author Sch2.

In Section 7, we consider examples of quantization of Belavin-Drinfeld triples.

2. Generalized Belavin-Drinfeld triples and solutions OF THE CLASSICAL DYNAMICAL YANG-BAXTER EQUATION

2.1. Notations on semisimple Lie algebras. Let $\mathfrak{g}$ be a semisimple Lie algebra over $\mathbb{C}$, and $\mathfrak{h} \subset \mathfrak{g}$ a Cartan subalgebra. Let $\Gamma \subset \mathfrak{h}^{*}$ be a set of simple roots of $\mathfrak{g}$. Fix a nondegenerate invariant inner product on $\mathfrak{g}$ which is positive definite on the real span of roots. We can identify $\mathfrak{h}$ with $\mathfrak{h}^{*}$ using the inner product. Let us denote by $h_{\alpha}$ the image of a root $\alpha$ under this identification.

Let $\mathfrak{n}_{ \pm}$be the positive and negative nilpotent subalgebras of $\mathfrak{g}$. We have a decomposition $\mathfrak{n}_{ \pm}=\bigoplus_{ \pm \alpha>0} \mathfrak{g}_{\alpha}$, where $\mathfrak{g}_{\alpha}$ are root subspaces of $\mathfrak{g}$. Let $e_{\alpha}$ be a generator of the root subspace $\mathfrak{g}_{\alpha}$ for any $\alpha>0$, and let $f_{\alpha}$ be a generator of $\mathfrak{g}_{-\alpha}$ such that $\left(e_{\alpha}, f_{\alpha}\right)=1$.

\subsection{Generalized Belavin-Drinfeld triples and dynamical r-matrices.}

Definition 2.1 ( $\mathrm{BD},[\mathrm{S}])$. A generalized Belavin-Drinfeld triple $\left(\Gamma_{1}, \Gamma_{2}, T\right)$ for $\mathfrak{g}$ consists of subsets $\Gamma_{1}, \Gamma_{2} \subset \Gamma$ together with a bijection $T: \Gamma_{1} \rightarrow \Gamma_{2}$ which preserves the inner product. If, in addition, $\forall \alpha \in \Gamma_{1}, \exists k \in \mathbb{N}$ such that $T^{k} \alpha \notin \Gamma_{1}$, the triple is said to be a nilpotent or ordinary Belavin-Drinfeld triple.

Given a generalized Belavin-Drinfeld triple, we define a Lie algebra homomorphism $T: \mathfrak{n}_{+} \rightarrow \mathfrak{n}_{+}$, by setting on simple root elements: $T\left(e_{\alpha}\right)=e_{T \alpha}$ if $\alpha \in \Gamma_{1}$, and zero otherwise. It is easy to see that such a homomorphism is well defined.

For any generalized Belavin-Drinfeld triple, let $\mathfrak{l} \subset \mathfrak{h}$ be the subspace defined by $\mathfrak{l}=\operatorname{Span}\left(\alpha-T \alpha \mid \alpha \in \Gamma_{1}\right)^{\perp}$.

It is clear that $\mathfrak{l}$ is a nondegenerate subspace for the inner product, since the inner product is positive definite on the real span of roots. 
Let us define a useful linear operator on the orthogonal complement $\mathfrak{l}^{\perp}$ to $\mathfrak{l}$ in $\mathfrak{h}$. To do this, observe that for any $x \in \mathfrak{l}^{\perp}$, there exists a unique $y \in \mathfrak{l}^{\perp}$ such that for all $\alpha \in \Gamma_{1}$ one has $(\alpha-T \alpha, y)=(\alpha+T \alpha, x)$. It is clear that $y$ depends linearly on $x$. We will write $y=C_{T} x$. It is easy to check that the operator $C_{T}: \mathfrak{l}^{\perp} \rightarrow \mathfrak{l}^{\perp}$ is skewsymmetric. This operator is called the Cayley transform of $T$.

To any generalized Belavin-Drinfeld triple $\left(\Gamma_{1}, \Gamma_{2}, T\right)$, one may associate a solution of the classical dynamical Yang-Baxter equation as follows.

For a vector space $V$ with a nondegenerate inner product, denote by $\Omega_{V}$ the element of $S^{2} V$ which is inverse to the inner product of $V$.

Define the function $r_{T}: \mathfrak{l}^{*} \rightarrow(\mathfrak{g} \otimes \mathfrak{g})^{\mathfrak{l}}$ by

$$
r_{T}(\lambda)=r-\frac{1}{2}\left(C_{T} \otimes 1\right) \Omega_{\mathfrak{l} \perp}+a(\lambda)-a^{21}(\lambda),
$$

where $r:=\frac{1}{2} \Omega_{\mathfrak{h}}+\sum_{\alpha} e_{\alpha} \otimes f_{\alpha}$ is the standard Drinfeld r-matrix and

$$
a(\lambda)=\sum_{\alpha} \sum_{l=1}^{\infty} e^{-l(\lambda, \alpha)} T^{l}\left(e_{\alpha}\right) \otimes f_{\alpha} .
$$

Proposition $2.1([\underline{\mathrm{S}}])$. The function $r_{T}(\lambda)$ is a solution of the classical dynamical Yang-Baxter equation

$$
\begin{aligned}
\sum_{i} & \left(x_{i}^{(1)} \frac{\partial}{\partial x_{i}} r^{23}(\lambda)-x_{i}^{(2)} \frac{\partial}{\partial x_{i}} r^{13}(\lambda)+x_{i}^{(3)} \frac{\partial}{\partial x_{i}} r^{12}(\lambda)\right) \\
& +\left[r^{12}(\lambda), r^{13}(\lambda)\right]+\left[r^{13}(\lambda), r^{23}(\lambda)\right]+\left[r^{12}(\lambda), r^{23}(\lambda)\right]=0 .
\end{aligned}
$$

Remark 2.1. In the expression for $r_{T}(\lambda)$, the sum $\sum_{l=1}^{\infty} e^{-l(\lambda, \alpha)} T^{l}\left(e_{\alpha}\right) \otimes f_{\alpha}$ is finite if $T$ acts nilpotently on $\alpha$, and is an infinite series convergent to a rational function of $e^{(\lambda, \alpha)}$ if a suitable power of $T$ preserves $\alpha$.

This proposition has a straightforward generalization to the case of semisimple Lie algebras.

2.3. The classification of dynamical $\mathbf{r}$-matrices. It is clear that $r_{T}+r_{T}^{21}=\Omega_{\mathfrak{g}}$. Conversely, it was shown in $[\mathrm{S}]$ that if $\mathfrak{g}$ is simple, then solutions $r_{T}(\lambda)$ exhaust all solutions of the classical dynamical Yang-Baxter equation with this property, up to isomorphism and gauge transformations. More precisely, the result is as follows.

Let $H$ be the Cartan subgroup of the Lie group $G$ corresponding to $\mathfrak{g}$, whose Lie algebra is $\mathfrak{h}$. By a gauge transformation of $\mathbf{r}: \mathfrak{l}^{*} \rightarrow(\mathfrak{g} \otimes \mathfrak{g})^{\mathfrak{l}}$, we mean a transformation

$$
\mathbf{r} \mapsto \mathbf{r}^{g}:=(\operatorname{Ad} g \otimes \operatorname{Ad} g)\left(\mathbf{r}-\left(g^{-1} d g\right)+\left(g^{-1} d g\right)^{21}\right)
$$

for $g: \mathfrak{l}^{*} \rightarrow H$ a meromorphic function (here the differential 1 -form $g^{-1} d g$ on $\mathfrak{l}^{*}$ with values in $\mathfrak{h}$ is regarded as a function on $\mathfrak{l}^{*}$ with values in $\left.\mathfrak{l} \otimes \mathfrak{h}\right)$. Now, we have the following theorem:

Theorem 2.1 ( $[\mathrm{S}])$. Let $\mathfrak{g}$ be simple, and let $\mathfrak{l}^{\prime} \subset \mathfrak{h}$ be any subalgebra which is nondegenerate with respect to the inner product on $\mathfrak{h}$. Let $\mathbf{r}: \mathfrak{l}^{\prime^{*}} \rightarrow(\mathfrak{g} \otimes \mathfrak{g})^{\mathfrak{l}^{\prime}}$ be any solution of the classical dynamical Yang-Baxter equation satisfying $\mathbf{r}+\mathbf{r}^{21}=\Omega_{\mathfrak{g}}$. Then $\mathbf{r}$ may be obtained from $r_{T}(\lambda)$ for a suitable triple by an automorphism of $\mathfrak{g}$ and a gauge transformation. 
The Belavin-Drinfeld result for the ordinary classical Yang-Baxter equation (CYBE) $\mathrm{BD}$ is easily obtained from the above classification. Namely, any solution $\mathbf{r} \in \mathfrak{g} \otimes \mathfrak{g}$ to the CYBE such that $\mathbf{r}+\mathbf{r}^{21}=\Omega_{\mathfrak{g}}$ is equivalent under an automorphism of $\mathfrak{g}$ to a solution of the form

$$
r_{T, s}=r-s+\sum_{\alpha} \sum_{l=1}^{\infty} T^{l}\left(e_{\alpha}\right) \wedge f_{\alpha}
$$

for an ordinary (nilpotent) Belavin-Drinfeld triple $\left(\Gamma_{1}, \Gamma_{2}, T\right)$, where $s \in \Lambda^{2} \mathfrak{h}$ is a solution of the equations

$$
((\alpha-T \alpha) \otimes 1)(2 s)=((\alpha+T \alpha) \otimes 1) \Omega_{\mathfrak{h}}, \forall \alpha \in \Gamma_{1} .
$$

This solution $r_{T, s}$ (which is independent of $\lambda \in \mathfrak{l}^{*}$ ) can be obtained by a gauge transformation from $r_{T}(\lambda)$.

Remark 2.2. Solutions of equation (2.6) always exist and form an affine space of dimension $\left(\Gamma-\left|\Gamma_{1}\right|\right)\left(\Gamma-\left|\Gamma_{1}\right|-1\right) / 2$ (see $\left.[\mathrm{BD}]\right)$.

Remark 2.3. It is clear from the definition of $C_{T}$ that, if regarded as an element of $\Lambda^{2} \mathfrak{l}^{\perp}$, it can be defined as the unique solution $C$ of the equation

$$
((\alpha-T \alpha) \otimes 1)(C)=((\alpha+T \alpha) \otimes 1) \Omega_{\Upsilon_{\perp}, \forall \alpha \in \Gamma_{1},}
$$

which is similar to (but not the same as) equation (2.6) for $2 s$.

Remark 2.4. In general, there is no simple closed form for $s$ in terms of $T$. This demonstrates yet another advantage of dynamical r-matrices: the dynamical $r$ matrix (2.1) has a simpler diagonal part than the (gauge equivalent to it) usual r-matrix (2.5).

Remark 2.5. It is not difficult to generalize the results of this section to the case of semisimple Lie algebras.

\section{The Dynamical twist $\mathcal{J}_{T}$}

3.1. The extension of $T$ to an orthogonal automorphism of $\mathfrak{l}$. The goal of this section is to construct a twist which provides a quantization of the classical dynamical r-matrices of Section 2 In order to define this twist, we need to extend the map $T$ to a linear map from $\mathfrak{h}$ to $\mathfrak{h}$.

Fix a finite dimensional semisimple Lie algebra $\mathfrak{g}$ and a generalized BelavinDrinfeld triple $\left(\Gamma_{1}, \Gamma_{2}, T\right)$. Let $\Gamma_{3} \subset \Gamma_{1}$ be the largest $T$-invariant subset of $\Gamma_{1}$. Set $\mathfrak{h}_{i}=\operatorname{Span}\left(\Gamma_{i}\right)$ for $i \in\{1,2,3\}$. It is clear that $T$ extends naturally to a linear map $T: \mathfrak{h}_{1} \rightarrow \mathfrak{h}_{2}$, which we will also denote by $T$.

Lemma 3.1. One has $\mathfrak{h}_{1} \cap \mathfrak{l}=\mathfrak{h}_{3}^{T}$. Moreover, $\mathfrak{h}_{1}+\mathfrak{l}=\mathfrak{h}$.

Proof. Let $x \in \mathfrak{h}_{1} \cap \mathfrak{l}$ be a real element. Then $(T x, T x)=(x, x)=(x, T x)=(T x, x)$. Thus, $(x-T x, x-T x)=0$, so the positive definiteness of the form on real elements implies $x=T x$. Since $T$ is nilpotent as a map $\Gamma_{1} \backslash \Gamma_{3} \rightarrow \Gamma_{2} \backslash \Gamma_{3}$, we get $x \in \mathfrak{h}_{3}^{T}$. This proves the first statement.

To prove the second statement, it is enough to notice that the rank of the system of linear equations $\alpha(y)=T \alpha(y), \alpha \in \Gamma_{1}$ (with respect to $y \in \mathfrak{h}$ ), is $\left|\Gamma_{1}\right|-\left|\Gamma_{3} / T\right|$. Thus, $\operatorname{dim}(\mathfrak{l})=|\Gamma|-\left|\Gamma_{1}\right|+\left|\Gamma_{3} / T\right|$. So the second statement follows from the first statement. 
Corollary 3.1. There is a unique extension $T: \mathfrak{h} \rightarrow \mathfrak{h}$ of $T: \mathfrak{h}_{1} \rightarrow \mathfrak{h}_{2}$ which is equal to the identity on $\mathfrak{l}$. This extension is an orthogonal operator.

Proof. By the lemma, $\mathfrak{h}=\mathfrak{h}_{1}+\mathfrak{l}$ and $T$ is the identity on $\mathfrak{h}_{1} \cap \mathfrak{l}$, which immediately implies that $T$ admits a unique extension as desired. The fact that the obtained extension is orthogonal is straightforward to verify.

3.2. The construction of the twist. Let $q=e^{\hbar / 2}$, where $\hbar$ is a formal parameter, and let $U_{q}(\mathfrak{g})$ be the Drinfeld-Jimbo quantum universal enveloping algebra, which is a quantization of the Lie bialgebra $(\mathfrak{g}, r)$ (see [CP, p. 281). Let $\mathcal{R}=1+\hbar r+\ldots$ be its universal R-matrix. We choose the coproduct of $U_{q}(\mathfrak{g})$ in such a way that the "Cartan part" of the R-matrix is $q^{\Omega_{\mathfrak{h}}}$.

Our goal in this subsection is to introduce a dynamical twist $\mathcal{J}_{T}(\lambda), \lambda \in \mathfrak{l}^{*}$, in the (completed) tensor square of $U_{q}(\mathfrak{g})$ (i.e. a solution of the dynamical 2cocycle condition) which would twist the universal R-matrix of $U_{q}(\mathfrak{g})$ to a matrix $R_{T}=1+\hbar r_{T}+O\left(\hbar^{2}\right)$.

Define the degree of an element of $U_{q}(\mathfrak{g})$ by $\operatorname{deg}\left(e_{i}\right)=1, \operatorname{deg}\left(f_{i}\right)=-1, \operatorname{deg}\left(h_{i}\right)=$ 0 , and $\operatorname{deg}(x y)=\operatorname{deg}(x)+\operatorname{deg}(y)$.

Let $U_{+}$denote the Hopf subalgebra of $U_{q}(\mathfrak{g})$ generated by elements $h_{\alpha}, e_{i}$, and let $U_{-}$denote the Hopf subalgebra of $U_{q}(\mathfrak{g})$ generated by elements $h_{\alpha}, f_{i}$.

Define Hopf algebra homomorphisms $T: U_{+} \rightarrow U_{+}, T^{-1}: U_{-} \rightarrow U_{-}$as follows: $T^{ \pm 1}$ on $\mathfrak{h}$ is defined as in Section 3.1, $T\left(e_{\alpha}\right)=e_{T \alpha}$ if $\alpha \in \Gamma_{1}, T\left(e_{\alpha}\right)=0$ for other simple roots $\alpha, T^{-1}\left(f_{\alpha}\right)=f_{T^{-1} \alpha}$ if $\alpha \in \Gamma_{2}, T^{-1}\left(f_{\alpha}\right)=0$ on other simple roots. It is easy to show that such homomorphisms exist and are unique.

Set $Z=\left(\frac{1}{2}\left(C_{T}-1\right) \otimes 1\right) \Omega_{\Upsilon_{\perp}}$.

Let $W_{2}$ be the vector space of rational functions of $e^{(\lambda, \alpha)}$ (for simple roots $\alpha$ ) with values in $\left(U_{+} \otimes U_{-}\right)^{\mathfrak{l}}$, where $\otimes$ is the completed tensor product in the $\hbar$-adic topology.

Remark 3.1. We use the notation $W_{2}$ since this space consists of 2-component tensors.

Define a linear operator $A_{L}^{2}: W_{2} \rightarrow W_{2}$ as follows:

$$
A_{L}^{2} X=\left(T \operatorname{Ad} e^{-\lambda} \otimes 1\right)\left(\mathcal{R} X q^{-\Omega_{\imath}}\right) .
$$

Remark 3.2. The notation $A_{L}^{2}$ has the following motivation: this is an operator on 2-component tensors, which applies $T$ to the left component (so $L$ stands for "left").

Let $I_{ \pm}$denote the kernels of the projections of $U_{ \pm}$to elements of zero degree.

The construction of the dynamical twist depends on the following proposition.

Proposition 3.1. There exists a unique element $\mathcal{J}_{T} \in W_{2}$ such that

1) $\mathcal{J}_{T}-q^{Z} \in I_{+} \otimes I_{-}$,

2) $\mathcal{J}_{T}$ satisfies the "modified ABRR equation", $A_{L}^{2} \mathcal{J}_{T}=\mathcal{J}_{T}$.

Remark 3.3. If $T=i d$, this proposition is contained in the paper ABRR, which motivates the terminology "the modified ABRR equation".

Proof. The statement is equivalent to the claim that there exists a unique $X_{0}$ belonging to $1+\left(I_{+} \otimes I_{-}\right)^{\mathfrak{l}}$ such that

$$
X_{0}=\left(T \operatorname{Ad} e^{-\lambda} \otimes 1\right)\left(\mathcal{R} X_{0} q^{-\Omega_{\mathfrak{h}}}\right)
$$


(then $\mathcal{J}_{T}=X_{0} q^{Z}$ ). Let us write $X_{0}$ as $1+\sum_{j \geq 1} X_{0}^{j}$, where $X_{0}^{j}$ are the terms of degree $j$ in the first component. Then the above equation can be written as a system of equations labeled by degree $j \geq 1$ :

$$
X_{0}^{j}=\left(T \operatorname{Ad} e^{-\lambda} \otimes 1\right)\left(q^{\Omega_{\mathfrak{h}}} X_{0}^{j} q^{-\Omega_{\mathfrak{h}}}\right)+\ldots,
$$

where $\ldots$ stands for terms that involve $X_{0}^{i}$ for $i<j$. (The zero degree equation is obviously satisfied, so we don't need to include it.) It is obvious that the operator

$$
1-\left(T \operatorname{Ad} e^{-\lambda} \otimes 1\right) \operatorname{Ad} q^{\Omega_{\mathfrak{h}}}
$$

on $W_{2}$ is invertible for generic $\lambda$. Thus, the last equation admits a unique solution for all $j$, which allows one to compute $X_{0}^{j}$ recursively. The proposition is proved.

3.3. The main theorem. The main theorem of this paper is the following:

Theorem 3.1. The element $\mathcal{J}_{T}$ satisfies the dynamical cocycle condition

$$
\mathcal{J}_{T}^{12,3}(\lambda) \mathcal{J}_{T}^{12}\left(\lambda-\frac{1}{2} \hbar h^{(3)}\right)=\mathcal{J}_{T}^{1,23}(\lambda) \mathcal{J}_{T}^{23}\left(\lambda+\frac{1}{2} \hbar h^{(1)}\right) .
$$

Here, by $\mathcal{J}_{T}^{12,3}$ we mean $(\Delta \otimes 1)\left(\mathcal{J}_{T}\right)$ where $\Delta: U_{q}(\mathfrak{g}) \rightarrow U_{q}(\mathfrak{g}) \otimes U_{q}(\mathfrak{g})$ is the coproduct of $U_{q}(\mathfrak{g})$ and similarly $\mathcal{J}_{T}^{1,23}=(1 \otimes \Delta)\left(\mathcal{J}_{T}\right)$, and $\lambda-\frac{1}{2} \hbar h^{(3)}$ is defined as follows. If $y_{1}, \ldots, y_{r}$ is a basis of $\mathfrak{l}$, and $\lambda=\left(\lambda^{1}, \ldots, \lambda^{r}\right)$ is the coordinate representation of $\lambda$ with respect to this basis, then $\lambda-\frac{1}{2} \hbar h^{(3)}=\left(\lambda^{1}-\frac{1}{2} \hbar y_{1}^{(3)}, \ldots, \lambda^{r}-\frac{1}{2} \hbar y_{r}^{(3)}\right)$, and for any meromorphic function $S(\lambda)$ we set $S\left(\lambda-\frac{1}{2} \hbar h^{(3)}\right)=S(\lambda)-\frac{1}{2} \hbar \sum_{i} \frac{\partial S}{\partial y^{i}}(\lambda) y_{i}+\ldots$ (the Taylor expansion). The definition of $\lambda+\frac{1}{2} \hbar h^{(1)}$ is similar.

The proof of the theorem is given in Section 4

Now define

$$
R_{T}(\lambda):=\left(\mathcal{J}_{T}^{21}\right)^{-1}(\lambda) \mathcal{R} \mathcal{J}_{T}(\lambda)
$$

Corollary 3.2. The function $R_{T}(\lambda)$ satisfies the (symmetrized) quantum dynamical Yang-Baxter equation

$$
\begin{aligned}
R_{T}^{12}\left(\lambda+\frac{1}{2} \hbar h^{(3)}\right) R_{T}^{13} & \left(\lambda-\frac{1}{2} \hbar h^{(2)}\right) R_{T}^{23}\left(\lambda+\frac{1}{2} \hbar h^{(1)}\right) \\
& =R_{T}^{23}\left(\lambda-\frac{1}{2} \hbar h^{(1)}\right) R_{T}^{13}\left(\lambda+\frac{1}{2} \hbar h^{(2)}\right) R_{T}^{12}\left(\lambda-\frac{1}{2} \hbar h^{(3)}\right) .
\end{aligned}
$$

The proof of the corollary is straightforward using the main theorem.

Remark 3.4. The symmetrized quantum dynamical Yang-Baxter equation was first considered by Felder. It is equivalent to the nonsymmetrized quantum dynamical Yang-Baxter equation

$$
R^{12}\left(\lambda-\hbar h^{(3)}\right) R^{13}(\lambda) R^{23}\left(\lambda-\hbar h^{(1)}\right)=R^{23}(\lambda) R^{13}\left(\lambda-\hbar h^{(2)}\right) R^{12}(\lambda),
$$

considered by many authors, by the change of variable $\lambda=-\left(\lambda^{\prime}+\frac{1}{2}\left(h^{(1)}+h^{(2)}\right)\right)$.

\subsection{The quasiclassical limit.}

Proposition 3.2. One has $R_{T}(\lambda)=1+\hbar r_{T}(\lambda)\left(\bmod \hbar^{2}\right)$, where $r_{T}(\lambda)$ is as given in the previous section. In other words, $R_{T}(\lambda)$ is a quantization of $r_{T}(\lambda)$. 
Proof. The quasiclassical limit of the modified ABRR equation has the form

$$
x=\left(T \operatorname{Ad} e^{-\lambda} \otimes 1\right)\left(r+x-\frac{1}{2} \Omega_{\mathfrak{l}}\right),
$$

where $x \in(\mathfrak{g} \otimes \mathfrak{g})^{\mathfrak{l}}$ has Cartan part $Z$. Solving this equation, we obtain that $\mathcal{J}_{T}=1+\hbar\left(\frac{Z}{2}+a\right)\left(\bmod \hbar^{2}\right)$. Since $\mathcal{R}=1+\hbar r\left(\bmod \hbar^{2}\right)$, the proposition is proved.

3.5. The nilpotent case. If the Belavin-Drinfeld triple is nilpotent, the element $\mathcal{J}_{T}$ can be written as a finite product, as follows.

For a nilpotent triple, let $n$ be the largest integer so that $T^{n}$ is defined on some $\alpha \in \Gamma_{1}$.

Proposition 3.3. In the nilpotent case, the solution $\mathcal{J}_{T}$ of the modified ABRR equation can be written in the form

$$
\mathcal{J}_{T}(\lambda)=\left(T \operatorname{Ad} e^{-\lambda} \otimes 1\right)(\mathcal{R}) \ldots\left(T^{n} \operatorname{Ad} e^{-n \lambda} \otimes 1\right)(\mathcal{R}) q^{Z-\left[\left(T+\ldots+T^{n}\right) \otimes 1\right]\left(\Omega_{\mathfrak{h}}\right)} .
$$

The proof of this proposition is obtained by substituting the modified ABRR equation into itself $n$ times.

3.6. The infinite product formula for $\mathcal{J}_{T}$. A product formula for $\mathcal{J}_{T}$ similar to the above exists for an arbitrary (not necessarily nilpotent) $T$, but in the nonnilpotent case the product is infinite. To write down the general formula, let $\mathcal{R}=$ $\mathcal{R}_{0} q^{\Omega_{\mathfrak{h}}}$, and

$$
\mathcal{R}_{0}^{m}(\lambda)=\operatorname{Ad} q^{\left(\left[T+\ldots+T^{m-1}\right] \otimes 1\right) \Omega_{\mathfrak{h}}}\left(T^{m} \operatorname{Ad} e^{-m \lambda} \otimes 1\right) \mathcal{R}_{0} .
$$

Then we have

$$
\mathcal{J}_{T}(\lambda)=\left[\prod_{m=1}^{\infty} \mathcal{R}_{0}^{m}(\lambda)\right] q^{Z} .
$$

This product is clearly convergent in the topology of formal power series in $e^{-(\lambda, \alpha)}$. If $T$ is nilpotent, the product becomes finite and we get the formula from the previous section.

\section{Proof of the main theorem}

4.1. The right component version of the modified ABRR equation. To prove Theorem 3.1 we introduce a "right-component version" of the modified ABRR equation. Define the linear operator $A_{R}^{2}: W_{2} \rightarrow W_{2}$ by

$$
A_{R}^{2} X=\left(1 \otimes T^{-1} \operatorname{Ad} e^{\lambda}\right)\left(\mathcal{R} X q^{-\Omega_{\mathrm{r}}}\right) .
$$

Lemma 4.1. The operators $A_{L}^{2}$ and $A_{R}^{2}$ commute.

Proof. This follows immediately from the fact that $(T \otimes 1)(\mathcal{R})=\left(1 \otimes T^{-1}\right)(\mathcal{R})$, which is true because $T$ preserves the inner product on $\mathfrak{h}$.

Corollary 4.1. $\mathcal{J}_{T}$ is the unique solution to the system of equations $A_{R}^{2} X=$ $X, A_{L}^{2} X=X$ with $\mathcal{J}_{T}-q^{Z} \in\left(I_{+} \otimes I_{-}\right)^{\mathfrak{l}}$.

Proof. We have $A_{L}^{2} A_{R}^{2} \mathcal{J}_{T}=A_{R}^{2} A_{L}^{2} \mathcal{J}_{T}=A_{R}^{2} \mathcal{J}_{T}$ so that $A_{R}^{2} \mathcal{J}_{T}$ and $\mathcal{J}_{T}$ are both solutions to $A_{L}^{2} X=X$ with zero degree term $q^{Z}$. Hence $\mathcal{J}_{T}=A_{R}^{2} \mathcal{J}_{T}$ by Proposition 3.1. 
4.2. The 3-component versions of the modified ABRR equation. Now, we introduce "3-component versions" of the modified ABRR equation. Let $W_{3}$ be the vector space of rational functions of $e^{(\lambda, \alpha)}$ for simple roots $\alpha$, with values in $\left(U_{+} \otimes U \otimes U_{-}\right)^{\mathfrak{l}}$, where $\otimes$ is the completed tensor product in the $\hbar$-adic topology.

Define linear operators $A_{L}^{3}, A_{R}^{3}: W_{3} \rightarrow W_{3}$ by

$$
\begin{aligned}
& A_{L}^{3} X=\left(T \operatorname{Ad} e^{-\lambda} \otimes 1 \otimes 1\right)\left(\mathcal{R}^{13} \mathcal{R}^{12} X q^{-\Omega_{\uparrow}^{13}-\Omega_{\uparrow}^{12}}\right), \\
& A_{R}^{3} X=\left(1 \otimes 1 \otimes T^{-1} \operatorname{Ad} e^{\lambda}\right)\left(\mathcal{R}^{13} \mathcal{R}^{23} X q^{-\Omega_{\uparrow}^{13}-\Omega_{\mathrm{\digamma}}^{23}}\right) .
\end{aligned}
$$

The 3-component versions of the modified ABRR equation are $A_{L}^{3} X=X, A_{R}^{3} X$ $=X$. They are obtained from the 2 -component equations by comultiplication of the component in which there is no action of $T$.

Lemma 4.2. The operators $A_{L}^{3}$ and $A_{R}^{3}$ commute.

Proof. This reduces to showing that

$$
\begin{aligned}
& \left(T \operatorname{Ad} e^{-\lambda} \otimes 1 \otimes 1\right)\left(\mathcal{R}^{13} \mathcal{R}^{12}\left(1 \otimes 1 \otimes T^{-1} \operatorname{Ad} e^{\lambda}\right)\left(\mathcal{R}^{13} \mathcal{R}^{23}\right)\right) \\
= & \left(1 \otimes 1 \otimes T^{-1} \operatorname{Ad} e^{\lambda}\right)\left(\mathcal{R}^{13} \mathcal{R}^{23}\left(T \operatorname{Ad} e^{-\lambda} \otimes 1 \otimes 1\right)\left(\mathcal{R}^{13} \mathcal{R}^{12}\right)\right) .
\end{aligned}
$$

Let $\overline{\mathcal{R}}=\left(T \operatorname{Ad} e^{-\lambda} \otimes 1\right) \mathcal{R}=\left(1 \otimes T^{-1} \operatorname{Ad} e^{\lambda}\right) \mathcal{R}$ and $\tilde{\mathcal{R}}=\left(T\right.$ Ad $\left.e^{-\lambda} \otimes T^{-1} \operatorname{Ad} e^{\lambda}\right) \mathcal{R}$. Then we need to check $\overline{\mathcal{R}}^{13} \overline{\mathcal{R}}^{12} \tilde{\mathcal{R}}^{13} \overline{\mathcal{R}}^{23}=\overline{\mathcal{R}}^{13} \overline{\mathcal{R}}^{23} \tilde{\mathcal{R}}^{13} \overline{\mathcal{R}}^{12}$, which follows (after cancelling the first factor) from the quantum Yang-Baxter equation for $\mathcal{R}$, applying $T \operatorname{Ad} e^{-\lambda}$ in the first component and $T^{-1} \mathrm{Ad} e^{\lambda}$ in the third one.

Lemma 4.3. If there exists a solution $X$ of $A_{L}^{3} X=A_{R}^{3} X=X$ such that $X-$ $q^{Z_{12}+Z_{13}+Z_{23}} \in I_{+} \otimes U(\mathfrak{g}) \otimes U(\mathfrak{g})+U(\mathfrak{g}) \otimes U(\mathfrak{g}) \otimes I_{-}$, then it is unique.

Proof. It is enough to show that such a solution $X$ is unique for the equation $A_{L}^{3} A_{R}^{3} X=X$. Let us make a change of variable $X=X_{0} q^{Z_{12}+Z_{13}+Z_{23}}$, and write $X_{0}=1+\sum_{k, l>0: k+l>0} X_{0}^{k, l}$, where $X_{0}^{k, l}$ is the part of $X_{0}$ having degree $k$ in the first component and $-l$ in the third component. It is easy to check that the equation for $A_{L}^{3} A_{R}^{3} X=X$ transforms to the system of equations

$$
X_{0}^{k, l}=\left(T \operatorname{Ad} e^{-\lambda} \otimes 1 \otimes T^{-1} \operatorname{Ad} e^{\lambda}\right)\left(q^{W} X_{0}^{k, l} q^{-W}\right)+\ldots, \quad k+l>0,
$$

where $W=\Omega_{\mathfrak{h}}^{12}+\Omega_{\mathfrak{h}}^{23}+[1 \otimes(1+T)] \Omega_{\mathfrak{h}}^{13}$, and ... stands for terms that involve $X_{0}^{k^{\prime}, l^{\prime}}$ with $k^{\prime}+l^{\prime}<k+l$ (the zero degree equation is obviously satisfied, so we don't need to include it). It is clear that the operator

$$
1-\left(T \operatorname{Ad} e^{-\lambda} \otimes 1 \otimes T^{-1} \operatorname{Ad} e^{\lambda}\right) \operatorname{Ad} q^{W}
$$

on $W_{3}$ is invertible for generic $\lambda$. Thus, the last equation admits a unique solution for all $k, l$, which allows one to compute $X_{0}^{k, l}$ recursively. The lemma is proved.

Now we complete the proof of the main theorem. It is obvious that $\mathcal{J}_{T}^{12,3}(\lambda)$. $\mathcal{J}_{T}^{12}\left(\lambda-\frac{1}{2} \hbar h^{(3)}\right)$ is a solution of $A_{R}^{3} X=X$ and that $\mathcal{J}_{T}^{1,23}(\lambda) \mathcal{J}_{T}^{23}\left(\lambda+\frac{1}{2} \hbar h^{(1)}\right)$ is a solution of $A_{L}^{3} X=X$. So, by virtue of the previous lemma, to prove the main theorem it is sufficient to prove the following.

Lemma 4.4. (i) $X=\mathcal{J}_{T}^{12,3}(\lambda) \mathcal{J}_{T}^{12}\left(\lambda-\frac{1}{2} \hbar h^{(3)}\right)$ is a solution of $A_{L}^{3} X=X$.

(ii) $X=\mathcal{J}_{T}^{1,23}(\lambda) \mathcal{J}_{T}^{23}\left(\lambda+\frac{1}{2} \hbar h^{(1)}\right)$ is a solution of $A_{R}^{3} X=X$. 
Proof. (i) As we mentioned, the element $X=\mathcal{J}_{T}^{12,3}(\lambda) \mathcal{J}_{T}^{12}\left(\lambda-\frac{1}{2} \hbar h^{(3)}\right)$ satisfies $A_{R}^{3} X=X$. Since $A_{L}^{3}$ and $A_{R}^{3}$ commute, the element $Y=A_{L}^{3} X$ is also a solution of $A_{R}^{3} X=X$. Since any element of $W_{3}$ invariant under $A_{R}^{3}$ is uniquely determined by its part of zero degree in the third component, it suffices to show that $A_{L}^{3} X$ has the same part of zero degree in the third component as $X$. Call the former $Y_{0}$ and the latter $X_{0}$. Clearly $X_{0}=q^{Z^{13}+Z^{23}} \mathcal{J}_{T}^{12}\left(\lambda-\frac{1}{2} \hbar h^{(3)}\right)$. Now, we find from $Y=A_{L}^{3} X$ that

$$
Y_{0}=\left(T \operatorname{Ad} e^{-\lambda} \otimes 1 \otimes 1\right)\left(q^{\Omega_{\mathfrak{h}}^{13}} \mathcal{R}^{12} q^{Z^{13}+Z^{23}} \mathcal{J}_{T}^{12}\left(\lambda-\frac{1}{2} \hbar h^{(3)}\right)\right) q^{-\Omega_{\mathfrak{r}}^{12}-\Omega_{\mathfrak{l}}^{13}}
$$

Since $\left[Z^{13}+Z^{23}, \mathcal{R}^{12}\right]=0$, we may rewrite this as

$$
Y_{0}=q^{Z^{23}}\left(T \operatorname{Ad} e^{-\lambda} \otimes 1 \otimes 1\right)\left(q^{\Omega_{\mathrm{I} \perp}^{13}+\Omega_{\mathrm{r}}^{13}+Z^{13}} \mathcal{R}^{12} \mathcal{J}_{T}^{12}\left(\lambda-\frac{1}{2} \hbar h^{(3)}\right)\right) q^{-\Omega_{\mathrm{r}}^{12}-\Omega_{\mathrm{l}}^{13}}
$$

Since $(T \otimes 1)\left(Z+\Omega_{\mathfrak{l} \perp}\right)=Z$, we have

$$
Y_{0}=q^{Z^{23}+Z^{13}}\left(T \operatorname{Ad} e^{-\lambda} \otimes 1 \otimes 1\right)\left(\operatorname{Ad} q^{\Omega_{\mathfrak{r}}^{13}}\left(\mathcal{R}^{12} \mathcal{J}_{T}^{12}\left(\lambda-\frac{1}{2} \hbar h^{(3)}\right)\right)\right) q^{-\Omega_{\mathrm{r}}^{12}}
$$

Next, note that $\operatorname{Ad} q^{h^{(3)}} \otimes 1 \otimes 1=\operatorname{Ad} q^{\Omega_{\mathrm{I}}^{13}}$, so that

$$
Y_{0}=q^{Z^{23}+Z^{13}}\left(T \operatorname{Ad} e^{-\lambda+\frac{1}{2} \hbar h^{(3)}} \otimes 1 \otimes 1\right)\left(\mathcal{R}^{12} \mathcal{J}_{T}^{12}\left(\lambda-\frac{1}{2} \hbar h^{(3)}\right)\right) q^{-\Omega_{\mathrm{\digamma}}^{12}} .
$$

Changing $\lambda$ to $\lambda-\frac{1}{2} \hbar h^{(3)}$ in the modified ABRR equation for $\mathcal{J}_{T}$, we see from the last equation that $Y_{0}=X_{0}$, as desired.

(ii) This is proved analogously to (i).

The main theorem is proved.

\section{QuANTIZATION OF GAUGE TRANSFORMATIONS}

In this section we provide a quantization for all gauge transformations, which yields a quantization for all dynamical r-matrices considered in Section 2 .

Let $g: \mathfrak{l}^{*} \rightarrow H$ be a meromorphic function.

Lemma 5.1. If $\mathcal{J}(\lambda) \in U_{q}(\mathfrak{g}) \otimes U_{q}(\mathfrak{g})$ is a solution of the dynamical cocycle condition of Theorem 3.1, then so is

$$
\mathcal{J}^{g}(\lambda)=(g(\lambda) \otimes g(\lambda)) \mathcal{J}(\lambda)\left(g^{-1}\left(\lambda-\frac{1}{2} \hbar h^{(2)}\right) \otimes g^{-1}\left(\lambda+\frac{1}{2} \hbar h^{(1)}\right)\right) .
$$

The proof of this lemma is straightforward.

Corollary 5.1. The element

$$
\mathcal{J}_{T}^{g}(\lambda)=(g(\lambda) \otimes g(\lambda)) \mathcal{J}_{T}(\lambda)\left(g^{-1}\left(\lambda-\frac{1}{2} \hbar h^{(2)}\right) \otimes g^{-1}\left(\lambda+\frac{1}{2} \hbar h^{(1)}\right)\right)
$$

satisfies the dynamical 2-cocycle condition. The element

$$
R_{T}^{g}(\lambda)=\left(\mathcal{J}_{T}^{g}\right)^{21}(\lambda)^{-1} \mathcal{R} \mathcal{J}_{T}^{g}(\lambda)
$$

satisfies the quantum dynamical Yang-Baxter equation, and is a quantization of the solution $r_{T}^{g}(\lambda)$ of the classical dynamical Yang-Baxter equation, which is obtained from $r_{T}$ by the gauge transformation $g$.

The proof of the corollary follows by an easy direct calculation. 


\section{The Nilpotent CASE}

6.1. The $\lambda$-independent twist. In the case of nilpotent (or ordinary) BelavinDrinfeld triples, the dynamical twist $\mathcal{J}_{T}(\lambda)$ may be transformed by a gauge transformation into an ordinary twist that does not depend on $\lambda$, and hence satisfies the ordinary (nondynamical) 2-cocycle condition

$$
\mathcal{J}^{12,3} \mathcal{J}^{12}=\mathcal{J}^{1,23} \mathcal{J}^{23} .
$$

This yields an explicit quantization for all nondynamical r-matrices defined in Section 2 .

Namely, in the setting of Section [5, set $g(\lambda)=e^{Q \lambda}$, where $Q: \mathfrak{l}^{*} \rightarrow \mathfrak{h}$ is a linear map. We have $\mathcal{J}_{T}^{g}(\lambda)=\left(\operatorname{Ad} e^{Q \lambda} \otimes \operatorname{Ad} e^{Q \lambda}\right) \mathcal{J}^{T}(\lambda) q^{Q^{21}-Q}$, where in the last factor we understand $Q$ as an element of $\mathfrak{l} \otimes \mathfrak{h}$. According to Section 5 , this element satisfies the dynamical 2-cocycle condition.

Now choose a solution $s$ of equation (2.6) and take $Q$ to be the component of $s$ in $\mathfrak{l} \otimes \mathfrak{l}^{\perp}$. Denote $\mathcal{J}_{T}^{g}$ by $\mathcal{J}_{T, s}$.

Theorem 6.1. $\mathcal{J}_{T, s}$ is independent of $\lambda$ (i.e. $\mathcal{J}_{T, s}: \mathfrak{l}^{*} \rightarrow U_{q}(\mathfrak{g}) \otimes U_{q}(\mathfrak{g})$ is constant). Hence, $\mathcal{J}_{T, s}$ satisfies the nondynamical 2-cocycle condition $\mathcal{J}^{12,3} \mathcal{J}^{12}=$ $\mathcal{J}^{1,23} \mathcal{J}^{23}$.

Proof. We may write as in Proposition 3.3 that

$$
\begin{array}{r}
\mathcal{J}_{T, s}=\left(\operatorname{Ad} e^{Q \lambda} \otimes \operatorname{Ad} e^{Q \lambda}\right)\left[\left(T \operatorname{Ad} e^{-\lambda} \otimes 1\right)(\mathcal{R}) \cdots\left(T^{n} \operatorname{Ad} e^{-n \lambda} \otimes 1\right)(\mathcal{R})\right. \\
\left.q^{Z-Q+Q^{21}-\left[\left(T+\ldots+T^{n}\right) \otimes 1\right]\left(\Omega_{\mathfrak{h}}\right)}\right] .
\end{array}
$$

Now, we note that

$$
\left(\operatorname{Ad} e^{Q \lambda} \otimes \operatorname{Ad} e^{Q \lambda}\right)\left(e_{T^{l} \alpha} \otimes f_{\alpha}\right)=e^{\left(Q \lambda, T^{l} \alpha-\alpha\right)} e_{T^{l} \alpha} \otimes f_{\alpha} .
$$

But by equation (2.6),

$$
\left(Q \lambda, T^{l} \alpha-\alpha\right)=\left(s, \lambda \otimes\left(T^{l} \alpha-\alpha\right)\right)=\sum_{j=0}^{l-1} \frac{1}{2}\left(\lambda,\left(T^{j}+T^{j+1}\right)(\alpha)\right)=l(\lambda, \alpha)
$$

$(\operatorname{as}(\lambda, T \alpha)=(\lambda, \alpha))$.

But it is easy to show from the ABRR equation that in the nilpotent case $\mathcal{J}_{T}$ is a linear combination with constant coefficients of products of $e^{-l(\lambda, \alpha)} e_{T^{l} \alpha} \otimes f_{\alpha}$ and elements from $U_{q}(\mathfrak{h})^{\otimes 2}$. Therefore, the above calculation precisely implies that there is no $\lambda$-dependence in $\mathcal{J}_{T, s}$.

\section{Corollary 6.1.}

$$
\mathcal{J}_{T, s}=(T \otimes 1)(\mathcal{R}) \ldots\left(T^{n} \otimes 1\right)(\mathcal{R}) q^{-s-\frac{1}{2} \Omega_{\mathfrak{I} \perp}-\left[\left(T+\ldots+T^{n}\right) \otimes 1\right]\left(\Omega_{\mathfrak{h}}\right)} .
$$

Proof. Since there is no $\lambda$-dependence, we can set $\lambda$ to 0 in the expression for $\mathcal{J}_{T, s}$, which after a short calculation yields the result.

Corollary 6.2. The element $R_{T, s}=\left(\mathcal{J}_{T, s}^{21}\right)^{-1} \mathcal{R} \mathcal{J}_{T, s}$ is a solution of the quantum Yang-Baxter equation $R^{12} R^{13} R^{23}=R^{23} R^{13} R^{12}$, which is a quantization of $r_{T, s}$.

The proof is straightforward. 
Remark 6.1. The fact that the element $J_{T, s}$ is a twist (i.e. satisfies the 2-cocycle condition) can be proved without ever mentioning "dynamical" objects, along the lines of Section 4. Namely, the new equations would be as before but with operators $\left(A_{L}^{2}\right)^{\prime},\left(A_{R}^{2}\right)^{\prime},\left(A_{L}^{3}\right)^{\prime},\left(A_{R}^{3}\right)^{\prime}$ defined as follows:

$$
\left(A_{L}^{3}\right)^{\prime} X=(T \otimes 1 \otimes 1)\left(\mathcal{R}^{13} \mathcal{R}^{12} X q^{-Q^{21}-Q^{31}+Q^{12}+Q^{13}}\right) q^{-Q^{12}-Q^{13}+Q^{21}+Q^{31}-\Omega_{\uparrow}^{12}-\Omega_{\uparrow}^{13},}
$$

$\left(A_{R}^{3}\right)^{\prime} X=\left(1 \otimes 1 \otimes T^{-1}\right)\left(\mathcal{R}^{13} \mathcal{R}^{23} X q^{-Q^{31}-Q^{32}+Q^{13}+Q^{23}}\right) q^{-Q^{13}-Q^{23}+Q^{31}+Q^{32}-\Omega_{\digamma}^{13}-\Omega_{\digamma}^{23}}$.

$$
\begin{gathered}
\left(A_{L}^{2}\right)^{\prime} X=(T \otimes 1)\left(\mathcal{R} X q^{Q-Q^{21}}\right) q^{Q^{21}-Q-\Omega_{\mathrm{l}}}, \\
\left(A_{R}^{2}\right)^{\prime} X=\left(1 \otimes T^{-1}\right)\left(\mathcal{R} X q^{Q-Q^{21}}\right) q^{Q^{21}-Q-\Omega_{\mathrm{l}}},
\end{gathered}
$$

These equations are obtained from the corresponding equations of Section 4 by the gauge transformation $e^{Q \lambda}$ as in Section 5, and then setting $\lambda$ to 0 .

One may show that $X=\mathcal{J}_{T, s}$ is the unique solution to $\left(A_{L}^{2}\right)^{\prime} X=X$ with a suitable part of zero degree in the first component, and also satisfies $\left(A_{R}^{2}\right)^{\prime} X=X$, and that $\left(\mathcal{J}_{T, s}\right)^{12,3}\left(\mathcal{J}_{T, s}\right)^{12}$ and $\left(\mathcal{J}_{T, s}\right)^{1,23}\left(\mathcal{J}_{T, s}\right)^{23}$ are both equal to the unique solution to the system $\left(A_{L}^{3}\right)^{\prime} Y=\left(A_{R}^{3}\right)^{\prime} Y=Y$ with a suitable zero degree part. This implies that $\mathcal{J}_{T, s}$ satisfies the 2-cocycle condition.

6.2. Explicit calculation of $\mathcal{J}_{T, s}$ in the vector representation for $\mathfrak{g}=\mathfrak{s l}_{n}$. Consider the case $\mathfrak{g}=\mathfrak{s l}(n)$. Let $\Gamma=\left\{\alpha_{1}, \ldots, \alpha_{n-1}\right\}$ be the set of simple roots where $\alpha_{i}=v_{i}-v_{i+1}$, and $v_{i}$ is the standard basis of $\mathbb{Z}^{n}$. For a root $\alpha=v_{i}-v_{j}$, let $e_{\alpha}=e_{i j}$ be the corresponding elementary matrix.

We have the $n$-dimensional representation $\phi: U_{q}(\mathfrak{g}) \rightarrow \operatorname{Mat}_{n}(\mathbb{C})$, given by $\phi\left(e_{\alpha_{i}}\right)=e_{i, i+1}, \phi\left(f_{\alpha_{i}}\right)=e_{i+1, i}, \phi\left(h_{\alpha_{i}}\right)=e_{i i}-e_{i+1, i+1}$. Let us calculate explicitly the matrix $(\phi \otimes \phi)\left(\mathcal{J}_{T, s}\right)$.

Let $\Gamma_{1}^{(k)} \subset \Gamma_{1}$ be the subset of all simple roots $\alpha$ on which $T^{k}$ is defined. Let $\tilde{\Gamma}_{1}^{(k)} \subset \operatorname{Span}\left(\Gamma_{1}^{(k)}\right)$ denote the subset of positive roots. Whenever $\alpha \in \tilde{\Gamma}_{1}^{(k)}$ is not simple, let $C_{\alpha, k}=1$ if $T^{k}$ reverses the orientation of $\alpha$ (as a segment on the Dynkin diagram) and 0 otherwise. For simple roots, let $C_{\alpha, k}$ be 0 .

Denote by $|\alpha|$ the number of simple roots in a positive root $\alpha$. For two positive roots $\alpha, \beta$ we will write $\alpha \lessdot \beta$ if $\alpha=v_{i}-v_{j}$ and $\beta=v_{j}-v_{k}$. Write $\alpha \prec \beta$ if $T^{k} \alpha=\beta$ for some $k>0$.

For $\alpha \prec \beta$, define

$$
L_{\alpha, \beta}=\frac{1}{2}[\alpha \lessdot \beta]-\frac{1}{2}[\beta \lessdot \alpha]+[\exists \gamma, \alpha \prec \gamma \prec \beta, \alpha \lessdot \gamma]-[\exists \gamma, \alpha \prec \gamma \prec \beta, \gamma \lessdot \alpha],
$$

where $[$ statement $]=0$ if statement is false and otherwise $[$ statement $]=1$.

Proposition 6.1. The action of $\mathcal{J}_{T, s}(\lambda)$ on the tensor product of two vector representations is given by

$$
(\phi \otimes \phi)\left(\mathcal{J}_{T, s}\right)=q^{-\frac{1}{2} \Omega_{\mathfrak{h}}} J_{1} \cdots J_{n} q^{-s+\frac{1}{2} \Omega_{\mathfrak{r}}},
$$

where

$$
J_{k}=1+\sum_{\alpha \in \tilde{\Gamma}_{1}^{(k)}}(-q)^{(|\alpha|-1) C_{\alpha, k}} q^{L_{\alpha, T^{k}}}\left(q-q^{-1}\right) e_{T^{k} \alpha} \otimes e_{-\alpha} .
$$


Proof. The proposition follows by a direct calculation from the explicit expression of the R-matrix in [KhT] and the above formula for $\mathcal{J}_{T, s}$.

Let

$$
R=q^{-1 / n}\left(q \sum_{i} e_{i i} \otimes e_{i i}+\sum_{i \neq j} e_{i i} \otimes e_{j j}+\left(q-q^{-1}\right) \sum_{i<j} e_{i j} \otimes e_{j i}\right)
$$

be the standard R-matrix in the vector representation (it is a quantization of $r$ in the vector representation).

Corollary 6.3. The element

$$
R(T, s)=q^{-s}\left(J_{n}^{21}\right)^{-1} \ldots\left(J_{1}^{21}\right)^{-1} R J_{1} \ldots J_{n} q^{-s}
$$

of $\operatorname{End}\left(\mathbb{C}^{n}\right) \otimes \operatorname{End}\left(\mathbb{C}^{n}\right)$ satisfies the quantum Yang-Baxter equation, and is a quantization of $r_{T}$ in the vector representation.

The proof is clear, noting that, in the vector representation, $\Omega_{\mathfrak{l}}$ commutes with anything that is invariant under $\mathfrak{l}$.

This corollary proves Part 1 of Conjecture 1.2 in [Sch2] since the element $R_{J}$ considered there is just $q^{\frac{1}{n}} R(T, s)^{21}$. The element $R_{J}$ coincides with the GGS $\mathrm{R}$-matrix in all checked cases, as detailed in $\mathrm{Sch} 2$.

\section{EXAmples of The TWist $\mathcal{J}_{T}$}

In this section we compute the twist $\mathcal{J}_{T}$ for $\mathfrak{g}=\mathfrak{s l}(n)$, evaluated in the representation $\phi: U_{q}(\mathfrak{g}) \rightarrow \operatorname{Mat}_{n}(\mathbb{C})$ in two particularly simple cases, when $\Gamma=\Gamma_{1}=\Gamma_{2}$. In particular, we give the twist for all $n$ where $T=i d$ (this is the case considered in $[\mathrm{ABRR}],[\mathrm{JKOS}]$ ), and for $\mathfrak{g}=\mathfrak{s l}(3)$ where $T\left(\alpha_{1}\right)=\alpha_{2}, T\left(\alpha_{2}\right)=\alpha_{1}$ (the "flip" map).

Proposition 7.1. For the triple $(\Gamma, \Gamma, i d)$ on $\mathfrak{s l}(n)$, one has

$$
(\phi \otimes \phi)\left(\mathcal{J}_{T}\right)=1+\sum_{i<j}\left(q-q^{-1}\right) \frac{1}{e^{\lambda_{j}-\lambda_{i}}-1} e_{i j} \otimes e_{j i},
$$

where $\lambda_{i}$ denotes the $i$-th entry of $\lambda$.

Proof. This can be computed by using the product formula

$$
\mathcal{J}_{T}=\prod_{m=1}^{\infty}\left(\operatorname{Ad} e^{-m \lambda} \otimes 1\right) \operatorname{Ad} q^{(m-1) \Omega_{\mathfrak{h}}}\left(\mathcal{R}_{0}\right)
$$

and evaluating in the vector representation, using the expression for $\mathcal{R}$ given in $\mathrm{KhT}$.

Proposition 7.2. For the "flip" triple $(\Gamma, \Gamma, T)$ where $\mathfrak{g}=\mathfrak{s l}(3)$, one has $(\phi \otimes \phi)\left(\mathcal{J}_{T}\right)$ $=J_{T} q^{Z}$, where

$$
\begin{aligned}
J_{T}(\mu)=1+\left(q-q^{-1}\right)[ & \frac{e^{-\mu}}{1-q^{-1} e^{-2 \mu}} e_{12} \otimes e_{32}+\frac{q e^{-2 \mu}}{1-q e^{-2 \mu}} e_{12} \otimes e_{21} \\
& +\frac{e^{-\mu}}{1-q e^{-2 \mu}} e_{23} \otimes e_{21}+\frac{q^{-1} e^{-2 \mu}}{1-q^{-1} e^{-2 \mu}} e_{23} \otimes e_{32} \\
& \left.+\frac{-q^{-1} e^{-2 \mu}+e^{-4 \mu}+q^{2} e^{-4 \mu}-q e^{-6 \mu}}{\left(1-e^{-4 \mu}\right)\left(1-q e^{-2 \mu}\right)} e_{13} \otimes e_{31}\right]
\end{aligned}
$$

letting $\mu$ denote $\lambda_{1}-\lambda_{2}=\lambda_{2}-\lambda_{3}$. 
Proof. This can be seen by expanding

$$
\mathcal{J}_{T} q^{-Z}=\prod_{m=1}^{\infty}\left(\operatorname{Ad} q^{\left[\left(T+\ldots+T^{m-1}\right) \otimes 1\right] \Omega_{\mathfrak{h}}}\left(T^{m} \operatorname{Ad} e^{-m \lambda} \otimes 1\right)\left(\mathcal{R}_{0}\right)\right),
$$

again using the formula for $\mathcal{R}$ found in [KhT].

One may use these formulas to explicitly compute the image of the twisted Rmatrix in the vector representation.

\section{ACKNOWLEDGEMENTS}

The work of P.E. was partially supported by NSF grant 9700477 , and was partly done when P.E. was an employee of the Clay Mathematical Institute as a CMI prize fellow. O.S. performed this research in part for the Clay Mathematical Institute. The work of T.S. was supported by the Harvard College Research Program. O.S. is grateful to the Harvard and MIT mathematics departments for their hospitality. The authors thank M. Gerstenhaber, A. Giaquinto, and T. Hodges for many useful discussions.

\section{REFERENCES}

[ABRR] Arnaudon, D., Buffenoir, E., Ragoucy, E., and Roche, Ph., Universal Solutions of quantum dynamical Yang-Baxter equations, Lett. Math. Phys. 44 (1998), no. 3, 201-214. MR 2000b:81058

[BD] Belavin, A.A., and Drinfeld, V.G., Triangle equations and simple Lie algebras. Soviet Sci. Rev. Sect. C: Math. Phys. Rev. 4 (1984), 93-165. MR 87h:58078

[CG] Cremmer, E., and Gervais, J.-L., The quantum group structure associated with non-linearly extended Virasoro algebras, Comm. Math. Phys. 134 (1990), 619-632. MR 92a: 81072

[CP] Chari, V., and Pressley, A., A guide to quantum groups, Cambridge University Press, Cambridge, 1994. MR 95j:17010; corrected reprint MR 96h:17014

[EK] Etingof, P., and Kazhdan, D., Quantization of Lie bialgebras I. Selecta Math. 2 (1996), no. 1, 1-41. MR 97f:17014

[ER] Etingof, P., and Retakh, V., Quantum determinants and quasideterminants, Asian Jour. Math. 3 (1999), no. 2, 345-352.

[ES] Etingof, P., and Schiffmann, O., Twisted traces of intertwiners for Kac-Moody algebras and classical dynamical r-matrices corresponding to generalized Belavin-Drinfeld triples, Math. Res. Lett. 6 (1999), no. 5-6, 593-613.

[EV] Etingof, P., and Varchenko, A., Exchange dynamical quantum groups, Comm. Math. Phys. 205 (1999), no. 1, 19-52. CMP 2000:01

[GGS] Gerstenhaber, M., Giaquinto, A., and Schack, S., Construction of quantum groups from Belavin-Drinfeld infinitesimals, in: Quantum deformations of algebras and their representations, Joseph, A., Shnider, S., editors, Israel Math. Conf. Proc. 7 (1993), 45-64. MR 94k:17022

[GH] Giaquinto, A., and Hodges, T. J., Nonstandard solutions of the Yang-Baxter equation, Lett. Math. Phys. 44 (1998), 67-75. MR 99h:81086

[H1] Hodges, T. J., The Cremmer-Gervais solutions of the Yang-Baxter equation, Proc. Amer. Math. Soc. 127 (1999), no. 6, 1819-1826. MR 99i:81102

[H2] Hodges, T. J., Generating functions for the coefficients of the Cremmer-Gervais Rmatrices, preprint, 1999.

[H3] Hodges, T. J., Nonstandard quantum groups associated to certain Belavin-Drinfeld triples, Perspectives on quantization (South Hadley, MA, 1996), 63-70, Contemp. Math., 214, Amer. Math. Soc., Providence, RI, 1998. MR 98k:17016

[JKOS] Jimbo, M., Konno, H., Odake, S., and Shiraishi, J., Quasi-Hopf twistors for elliptic quantum groups, Transform. Groups 4 (1999), no. 4, 303-327. CMP 2000:05

[KhT] Khoroshkin, S., and Tolstoy, V., Universal R-matrix for quantized (super)algebras. Comm. Math. Phys. 141 (1991), no. 3, 599-617. MR 93a:16031 
[S] Schiffmann, O., On classification of dynamical r-matrices, Math. Res. Lett. 5 (1998), 13-30. MR 99j:17026

[Sch1] Schedler, T., Verification of the GGS conjecture for $\mathfrak{s l}(n), n \leq 12$. Preprint, math.QA/9901079.

[Sch2] Schedler, T., On the GGS conjecture. Preprint, math.QA/9903079.

$[\mathrm{Xu}] \quad \mathrm{Xu}, \mathrm{P} .$, Quantum groupoids, math.QA 9905192, (1999).

Department of Mathematics, Room 2-165, Massachusetts Institute of Technology, 77 Massachusetts Avenue, Cambridge, Massachusetts 02139

E-mail address: etingof@math.harvard.edu

059 Pforzheimer House Mail Center, Cambridge, Massachusetts 02138

E-mail address: schedler@fas.harvard.edu

Department of Mathematics, Massachusetts Institute of Technology, 77 MassachuSetts Avenue, Cambridge, Massachusetts 02139

E-mail address: schiffma@clipper.ens.fr

Current address: Department of Mathematics, Yale University, New Haven, Connecticut 06520

E-mail address: schiffma@math.yale.edu 